\title{
INFORMATION THEORETIC MODELING OF PARANODAL REGIONS IN MYELINATED AXONS
}

\author{
Caglar Koca ${ }^{1}$, Ozgur Ergul ${ }^{2}$, Meltem Civas ${ }^{3}$, Ozgur B. Akan ${ }^{1,3}$ \\ ${ }^{1}$ Internet of Everthing (IoE) Group, Department of Engineering, University of Cambridge, UK , ${ }^{2}$ Gazi University, Faculty of \\ Engineering, Department of Electrical and Electronics Engineering, Ankara, Turkey, ${ }^{3}$ Next-generation and Wireless \\ Communications Laboratory (NWCL), Department of Electrical and Electronics Engineering, \\ Koç University, Istanbul, Turkey \\ NOTE: Corresponding author: Caglar Koca, ck542@cam.ac.uk
}

\begin{abstract}
As a natural form of nanoscale communication, neuro-spike communication inspires the deployment of nanomachines inside the human body for healthcare. To this end, the identification of failure mechanisms in normal and diseased connections of nervous nano-networks is crucial. Thus, in this paper, we investigate the information transmission through a single myelinated axon segment. We introduce a realistic multi-compartmental model for a single myelinated segment by incorporating the axon's paranodal regions to the model. Next, we characterize the myelinated segment communication channel in terms of attenuation over the range of frequencies. Based on this, we derive the rate per channel use and upper bound on the information capacity. The performance evaluations reveal that our approach provides dramatic correction regarding frequency response. We believe that this result could have a significant effect on the characterization of demyelinated axons from the information and communication technology (ICT) perspective.
\end{abstract}

Keywords - ICT-based treatment, intra-body nano-networks, Internet of Bio-Nano Things, demyelination, neuro-spike communication

\section{INTRODUCTION}

Advances in nanotechnology have opened the way for the deployment of nanomachines collaborating within the Internet of Bio-Nano Things (IoBNT) framework inside the human body as a new means of information and communication technology (ICT)-based treatment technique $[1,2]$. IoBNT offers both the abstraction necessary for a deeper look into the evolution of intra-body communications [3] and the foundation for analyzing and interacting with living organisms. For example, as a large scale intrabody nano-network, the human nervous system bears an enormous potential to inspire architectures for such systems with novel applications in diverse areas, including smart healthcare [4].

Shannon's information theory is usually applied to existing intra-body nano-networks to analyze intra-body communication mechanisms, one of which is neuro-spike communication achieved via molecules [5]. Neuro-spike communication consists of consecutive stages, namely, spike generation, axonal and synaptic transmission. Understanding of communication failures within any of these stages due to nervous system diseases such as spinal cord injury and Multiple Sclerosis (MS) is crucial to the designs of assistive or replacement nanomachines [6]. Accordingly, an accurate communication theoretical analysis of intra-body communication channels requires realistic channel modeling of healthy and diseased connections, which is promising in understanding disease mechanisms in biological systems communicating via molecules [7].
Regarding axonal transmission, communication and computational models of axons exist in the literature. Communication models mostly assume the axon as an ideal all-pass filter [8]. In biologically detailed communication models, the axon is modeled as a low-pass filter, and modified second-order Butterworth filters $[9,10]$. In more complex models, axonal propagation is represented with several state transitions in the case of hippocampal pyramidal neurons [11]. Moreover, an infinite transmission line model is also available [12]. The limitations of communication models include that they cannot fully capture the behavior of axons, which arises from the axon's physiology. The computational models, on the other hand, are accepted as biologically accurate. Thus, computational models can be used to construct the realistic channel model of axons, enabling accurate analyses. In this respect, multi-compartment models successfully describe the axonal transmission by dividing an axonal cable into compartments, where each compartment has the same membrane properties [13]. However, this approach neglects some parts of the axon, e.g., paranodal regions of myelinated axons, where the membrane properties are not uniform.

In many vertebrates, an insulating substance called myelin sheath surrounds axons. The myelin sheath is the extension of the plasma membrane of glial cells wrapping the axon, possibly in a multilayered structure. Myelin sheaths are formed in periodic segments. The short portions of the axon left uncovered by myelin are called the nodes of Ranvier. Axonal transmission is achieved by 

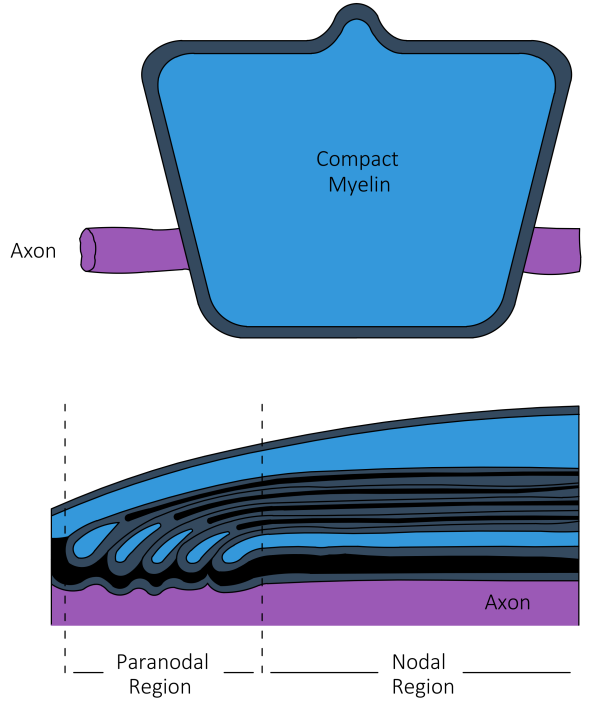

Fig. 1 - Paranodal and nodal regions of the myelin sheath.

impulse-like potential changes in the neuron membrane, called Action Potential (AP). Myelination increases the speed of signal propagation along the axon considerably by a process called saltatory conduction, which is simply the jumping of APs between the consecutive nodes of Ranvier. Successful saltatory conduction is strongly related to the structure and integrity of the myelin sheath [14]. In an intact and sufficiently thick sheath, ion leakage from the neuron membrane is minimal. As a result, attenuation at the membrane potential is also minimal. However, demyelination, which is the loss of myelin sheath, can increase ion leakage from the axon membrane to a level that the membrane potential attenuates too much when it reaches the next node. In this case, low membrane potential may not be sufficient to open $\mathrm{Na}^{+}$channels in the node, and consequently, AP propagation is blocked [15].

The myelin sheaths generally form in multiple layers. The sheath is wrapped around the axon starting from its short edge as shown in Fig.1. This structure of myelin forms intermediary regions called the paranodal regions between the nodes of Ranvier and the nodal regions of the myelin sheath. Assuming that the n-fold myelin sheath begins abruptly following a node of Ranvier causes inaccuracy when modeling the leakage resistance and capacitance of the region. Bearing in mind that there may be hundreds, even thousands of nodal and paranodal regions in a single axon, the importance of inaccuracy caused by oversimplistic modeling becomes apparent. Moreover, as shown in [16], even minor changes in the structure of paranodal regions can affect AP propagation significantly. This evidence shows that we should also consider paranodal regions to obtain a realistic model of myelinated axons.

In this paper, we propose a detailed model for the internode in a myelinated axon by taking paranodal regions into account, based on experimental evidence from the literature. Our aim is to investigate the frequency response properties of a single internodal channel. We perform frequency domain analysis of the system to quantify channel attenuation and compare the results with the classical cable model to show the effect of paranodal regions on information transmission. Then, we derive the rate per channel use and channel capacity when different forms of biological noise sources exist in the environment.

The rest of the paper is organized as follows. First, in Section 2, we describe the system model. Secondly, in Section 3, we define noise sources affecting the internodal channel and derive bounds on rate per channel use and capacity. Next, in Section 4, we provide numerical analysis of the channel's frequency domain properties. Finally, in Section 5, conclusions and future directions are summarized.

\section{SYSTEM MODEL}

A myelinated axon consists of active and passive compartments that sustain the active and passive spread of action potential through the axon, respectively. The nodes of Ranvier, which contain dense ion channels, are active compartments. In contrast, electrically neutral myelinated segments, i.e., internodes, with low ion channel density constitute passive compartments [17] as shown in Fig. 2. Due to the stochastic opening and closing of ion channels, which can be described via nonlinear differential equations of Hodgkin Huxley formalism, membrane resistance is time-varying at the nodes of Ranvier. On the other hand, since passive compartments have only a negligible number of ion channels, membrane resistance does not depend on time, and the axon acts linearly at internodes.

To investigate the linear response of an internode, we take paranodal regions into account. In this respect, classical cable theory, which is proven to be successful at explaining the behavior of AP propagation through the cylindrical structures such as dendrites and axons, is utilized [18].

\subsection{The cable equation}

According to cable theory, the membrane voltage is given by the following differential equation [13]

$$
\frac{1}{r_{f}} \frac{\partial^{2} V}{\partial x^{2}}=c_{m} \frac{\partial V}{\partial t}+\frac{V}{r_{m}},
$$

where $r_{m}, r_{f}$ and $c_{m}$ are the membrane resistance, forward resistance and membrane capacitance of the axon, respectively. Using the length constant, $\lambda=\sqrt{\frac{r_{m}}{r_{f}}}$ and the time constant, $\tau=r_{m} c_{m}$, Eq. (1) becomes

$$
\lambda^{2} \frac{\partial^{2} V}{\partial x^{2}}=\tau \frac{\partial V}{\partial t}+V
$$




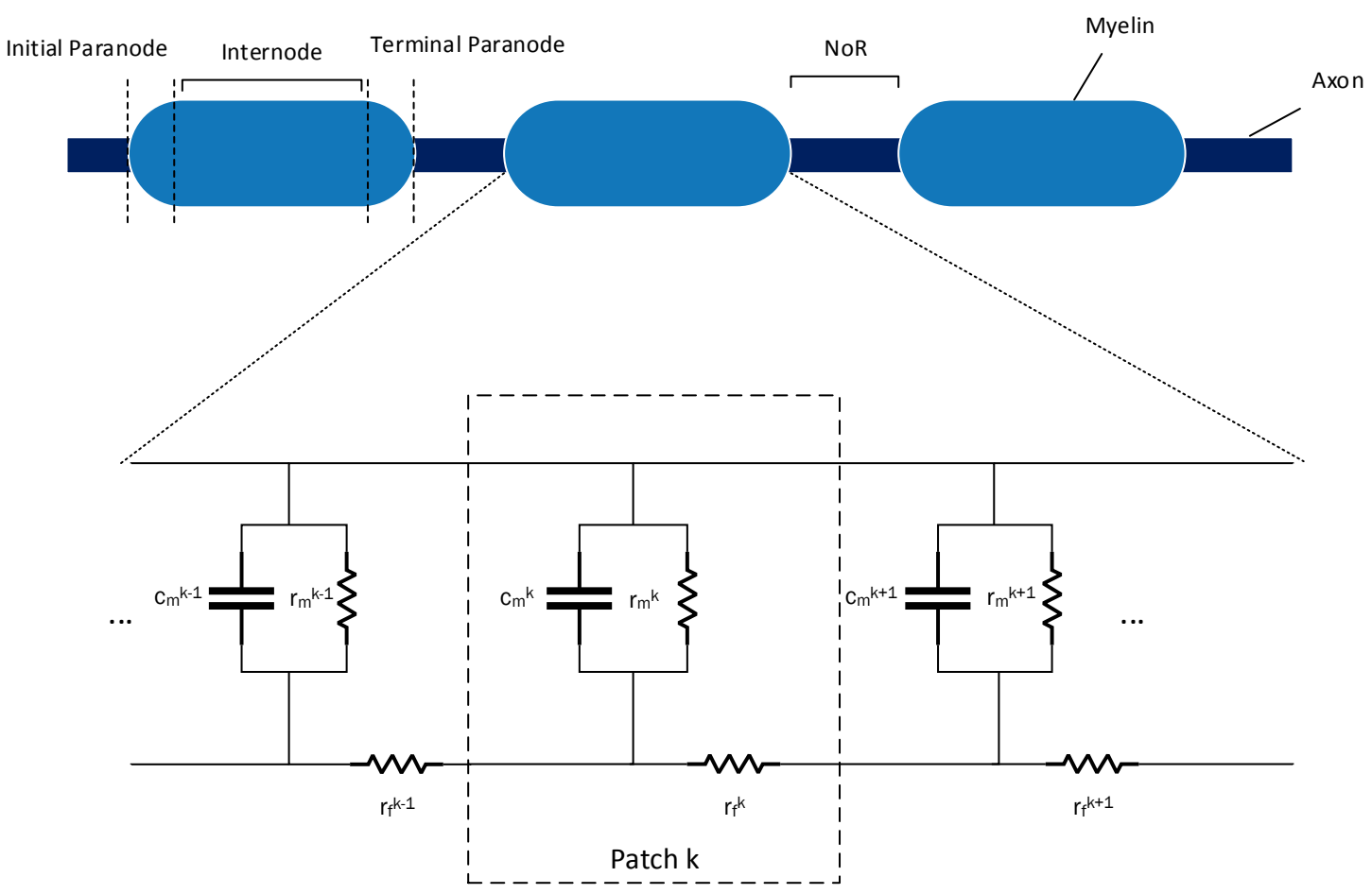

Fig. 2 - Two-dimensional diagram of myelinated axon and the circuit corresponding to a myelinated segment. The regions where axon is surrounded by myelin sheath are called internode and remaining parts without myelin are called Node of Ranvier (NoR). A myelinated segment consists of internodal region with constant myelin thickness, initial and terminal paranodes, where number of myelin wraps depends on the distance from and to NoR, respectively.

In the simplistic model of the myelinated axon, leakage resistance and capacitance are constant. However, due to the shape of the myelin sheath, paranodal regions do have different leakage resistance and capacitance values. Therefore, rather than taking $r_{m}$ and $c_{m}$ constant, we take them as a function of the distance from the start of the myelinated segment. Hence,

$$
\begin{aligned}
& \lambda \sim \lambda(x) \\
& \tau \sim \tau(x)
\end{aligned}
$$

The lumped circuit approximation, valid due to the small dimensions of the myelinated segments compared to the wavelengths of the dominant components forming the action potential, helps us to assume any field effect is instantaneous throughout the entire segment. Therefore, to calculate attenuation values in the steady state, partial derivative of voltage with respect to the time variable becomes zero, i.e., $\frac{\partial V}{\partial t}=0$. Hence, the resulting equation becomes

$$
V=\lambda^{2}(x) \frac{\mathrm{d}^{2} V}{\mathrm{~d} x^{2}}
$$

This is the inhomogeneous heat flow equation for the steady state [19]. Apart from rare simple cases, the cable equation cannot be solved analytically but numerically [13].
Since solving this equation is hard, we employ the fi- nite difference method, by partitioning our model into $N$ patches.

\subsection{The discrete axonal cable}

We consider a multi-compartment model of the myelinated axon to solve the inhomogeneous heat flow equation described in Section 2.1. Since this problem is hard to solve analytically, we attempt to solve this problem using the finite element method. By compartmentalizing the myelinated segment, where we assume the resistance and capacitance values are constant for the given compartment, we obtain a numerical solution to the heat flow problem.

Here, we divide a length- $l$ myelinated segment into $N$ compartments, where $N$ is a large integer [20]. Each of these pieces consists of three circuit elements, i.e., a forward resistance, $r_{f}$, leakage resistance, $r_{m}$ and leakage capacitance, $c_{m}$.

The equivalent circuit is shown in Fig. 2. Looking at Fig. 2 , we see the $(k-1)^{t h}, k^{t h}$ and $(k+1)^{t h}$ compartments of the myelinated segment, which we divided into $N$ compartments. The length of each compartment is $\Delta x=\frac{l}{N}$. Note that the smaller $\Delta x$ is, the more accurate the finite element method will be. 


\subsection{Multilayer cell membrane}

In the previous section, we described how the finite element method transforms the cable theory into a circuit with $N$ different compartments. In this section, we present how circuit parameters for each compartment are calculated. There are three circuit elements we need to investigate, namely, forward resistance, $r_{f}$, leakage resis- tance, $r_{m}$, and leakage capacitance, $c_{m}$.

\section{Forward resistance}

The forward resistance for the $k^{t h}$ compartment is obtained as

$$
r_{f}^{k}=R_{f} \frac{\Delta x}{2 \pi a}
$$

where $R_{f}$ is the specific forward resistance of the axon. Note that $r_{f}^{k}=r_{f} \forall k$, as forward resistance is only due to the cytoplasmic resistance of axon, not on the myelin covers spanning the axon.

\section{Leakage resistance}

We calculate the leakage resistance of an unmyelinated segment of thickness $\Delta x$ as

$$
r_{m}=\frac{R_{m}}{2 \pi a \Delta x}
$$

where $a$ is the axon radius and $R_{m}$ is the specific leakage resistance.

In order to calculate the leakage resistance of a myelinated segment, we first need to use the result in Eq. (7) to obtain the resistivity of axolemma, i.e.,

$$
\begin{aligned}
\rho & =\frac{r_{m}}{2 \pi a \Delta x} \frac{1}{\ln \left(\frac{a+d_{a}}{a}\right)} \\
& =\frac{R_{m}}{\ln \left(\frac{a+d_{a}}{a}\right)}
\end{aligned}
$$

where $d_{a}$ is the axolemma thickness and we assumed axolemma to be cylindrical.

In case axolemma is covered with multiple myelin turns, we can assume each turn to be cylindrical resistors with inner thickness $a+n \times d_{m}$ and outer thickness $a+d_{a}+$ $n \times d_{m}$ where $d_{m}$ is the myelin layer thickness. Note that myelin tissue shows remarkable similarities with the axolemma. Hence, we model myelin as axolemma encapsulating some intracranial fluid, where $d_{m}$ is the total layer thickness including the encapsulated intracranial fluid and the myelin itself, while $d_{a}$ is only the thickness of myelin, equal to the axolemma thickness. The crosssectional view of our model is depicted in Fig. 3. Since all

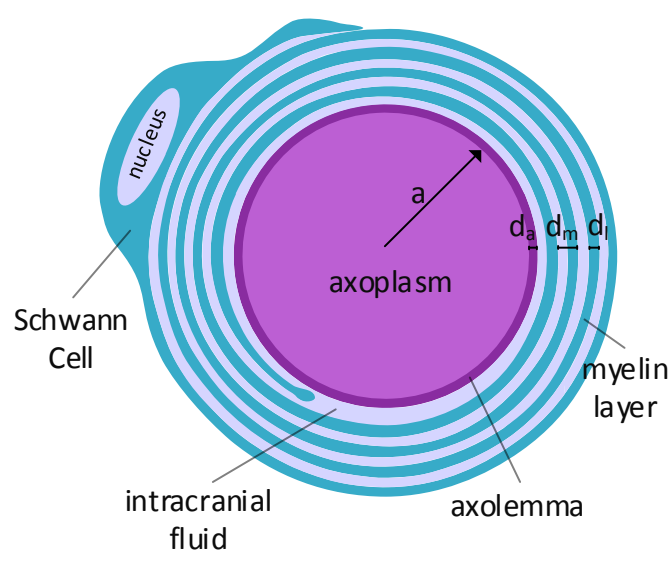

Fig. 3 - Cross-sectional view of myelinated axon.

myelin layers are connected in series, we can calculate the total axonal leakage resistance of a segment with length $\Delta x$ and myelin cover $n$ from axoplasm to the intracranial fluid as

$$
r_{m}=\sum_{0}^{n} \frac{\rho}{2 \pi \Delta x} \ln \left(\frac{a+d_{a}+n d_{m}}{a+n d_{m}}\right) .
$$

In the paranodal region, myelin turns wrapping a segment are not constant. If the last myelin layer is a partial turn, i.e., not covering the whole axolemma, we can still use Eq. (10) with a slight modification such that

$$
\begin{aligned}
r_{m}= & \sum_{0}^{\lfloor n\rfloor}\left(\frac{\rho}{2 \pi \Delta x} \ln \left(\frac{a+d_{a}+n d_{m}}{a+n d_{m}}\right)\right)+ \\
& (n-\lfloor n\rfloor) \frac{\rho}{2 \pi \Delta x} \ln \left(\frac{a+d_{a}+\lceil n\rceil d_{m}}{a+\lceil n\rceil d_{m}}\right)
\end{aligned}
$$

where the last part is due to the partial wrap. Note that here $n$ is not an integer. Rather, $n-\lfloor n\rfloor$ gives us the ratio of the partial cover.

Finally, we can calculate the leakage resistance of the $k^{t h}$ piece of a length $l$, radius $a$ and the paranodal region length $d$ myelinated segment with Eq. (11) by switching $n$ to $n^{k}$ as

$$
n^{k}= \begin{cases}\frac{N k \Delta x}{d}, & k \Delta x \leq d \\ \frac{N(d-k \Delta x)}{d}, & k \Delta x \geq l-d \\ N, & \text { e.w }\end{cases}
$$

\section{Leakage capacitance}

Here we can pursue an approach similar to leakage resistance. Leakage capacitance of an unmyelinated segment of thickness $\Delta x$ calculated as 


$$
c_{m}=C_{m} \Delta x(2 \pi a)
$$

Using Eq. (25), we can calculate electric permittivity of axolemma as

$$
\begin{aligned}
\epsilon & =\frac{c_{m}}{2 \pi \Delta x} \ln \left(\frac{a+d_{a}}{a}\right) \\
& =C_{m} a \ln \left(\frac{a+d_{a}}{a}\right) .
\end{aligned}
$$

Similar to the leakage resistance calculations, we can reach the leakage capacitance by modeling the myelin turns as concentric cylindrical capacitors with inner radii $a+n \times d_{m}$ and outer radii as $a+d_{a}+n \times d_{m}$. The resultant capacitance would be

$$
c_{m}=\frac{2 \pi \Delta x}{\sum_{0}^{n} \ln \left(\frac{a+d_{a}+n d_{m}}{a+n d_{m}}\right)} .
$$

We can calculate the leakage capacitance of a segment with length $\Delta x$ in case of partial myelin turns as

$$
c_{m}=\frac{2 \pi \Delta x}{\left(\sum_{0}^{\lfloor n\rfloor} \ln \left(\frac{a+d_{a}+\lfloor n\rfloor d_{m}}{a+\lfloor n\rfloor d_{m}}\right)\right)+\ln \frac{a+d_{a}+\lceil n\rceil d_{m}}{a+\lceil n\rceil d_{m}}} .
$$

Similar to the leakage resistance, we can find the leakage capacitance at the $k^{\text {th }}$ segment by using Eq. (18) with $n^{k}$ instead of $n$ as in Eq. (13).

\section{Outer resistance}

Outer resistance, or extracellular resistance, is the resistance of the fluid between axons [21]. Here, we assume that outer resistance is due to the extracellular fluid enclosed by the endoneurium.

We calculate the outer resistance similar to the forward resistance, i.e.,

$$
r_{o}^{k}=\frac{R_{o} \Delta x}{\pi\left(d_{e}^{2}-\left(d_{e}-d_{h}\right)^{2}\right)},
$$

where $R_{o}$ is the specific outer resistance, $d_{e}$ is the radius of the endoneurium and $d_{h}$ is the thickness of the endoneurium. Since endoneurium thickness and radius are accepted to be constant through the axon, $r_{o}^{k}$, similar to $r_{f}^{k}$, is independent of $k$.

\section{INTERNODAL CHANNEL CAPACITY}

In this section, we find the rate per channel use in internodal regions of myelinated axons.
Information transmission through axons is affected by noise sources including channel noise due to random fluctuations of voltage-gated ion channels at the node of Ranvier, thermal noise (Johnson noise) produced by membrane resistance, membrane capacitance, and cytoplasmic resistance, and crosstalk noise caused by axon-axon interactions such as ephaptic coupling $[22,23]$. Concerning the internodal channel, we do not consider channel and crosstalk noise, since the former is effective at the nodes of Ranvier and the latter is specific to neuron types [24]. Here, thermal noise is the only noise source that affects the internodal transmission in general; therefore, we use it to offer an upper bound for the per-use rate of the internodal channel. Thus, the power spectral density of thermal noise voltage is given by

$$
N_{P}(f)=2 k T \operatorname{Re}\{Z(f)\},
$$

where $k$ is the Boltzmann constant, $\mathrm{T}$ is the temperature in Kelvin and $Z$ is the total impedance of the system [23]. Since, $Z$ depends on frequency, thermal noise is also frequency dependent.

Action potential, the input to the channel, is represented by voltage variable $x(t)$. The power spectral density of signal $x(t)$ over the finite time interval $[0, T]$ is given by

$$
P(f)=\lim _{T \rightarrow \infty} \frac{1}{T}|X(f)|^{2},
$$

where $X(f)$ is the Fourier transform of $x(t)$. The input signal has power constraint that

$$
\int_{B} P(f) \leq P
$$

where $P$ is the average transmitted signal power over given bandwidth $B$.

Hence, the rate per channel use of a myelinated segment under thermal noise is bounded by

$$
R<\int_{-B / 2}^{B / 2} \log _{2}\left(1+\frac{|G(f)|^{2} P(f)}{N_{P}(f)}\right) \mathrm{d} f
$$

where $G(f)$ is the channel gain function and $B$ is channel bandwidth. The channel capacity of a myelinated segment can be obtained by water-filling. However, due to the frequency-dependent gain function, first, we need to calculate the effective noise spectral density, i.e.,

$$
N_{P}^{e f f}(f)=\frac{N_{P}(f)}{|G(f)|^{2}} .
$$

Thus, the capacity of the channel is found as

$$
C=\Delta_{f} \sum_{i=1}^{M} \log _{2}\left(1+\frac{P\left(f_{i}\right)}{N_{P}^{e f f}\left(f_{i}\right)}\right),
$$


where $M$ is the number of sub-bands with each having a width of $\Delta f$ that is sufficiently small. The power distribution that maximizes the capacity can be obtained by solving the Lagrange multiplier problem. Hence, the solution is given by [25]

$$
P(f)= \begin{cases}K-N_{P}^{e f f}(f) & f \in B \\ 0 & f \notin B\end{cases}
$$

where $K$ value satisfies (22).

\section{PERFORMANCE EVALUATION}

In this section, we first present the leakage resistance and capacitance trend graphically, and then the results of our frequency domain analysis of the internode.

The simulation environment for the results given in this section is Python. We choose Python over dedicated simulators such as Comsol or Neuron due to its flexibility in model building.

\subsection{Leakage resistance and capacitance}

Since the leakage impedance depends on the average number of layers, where each layer acts as another cell membrane, the leakage impedance in the paranodal region must increase linearly, as our model suggests. The difference between our model and the conventional model [18] is displayed in Fig. 4 and Fig. 5 for leakage resistance and leakage capacitance respectively.

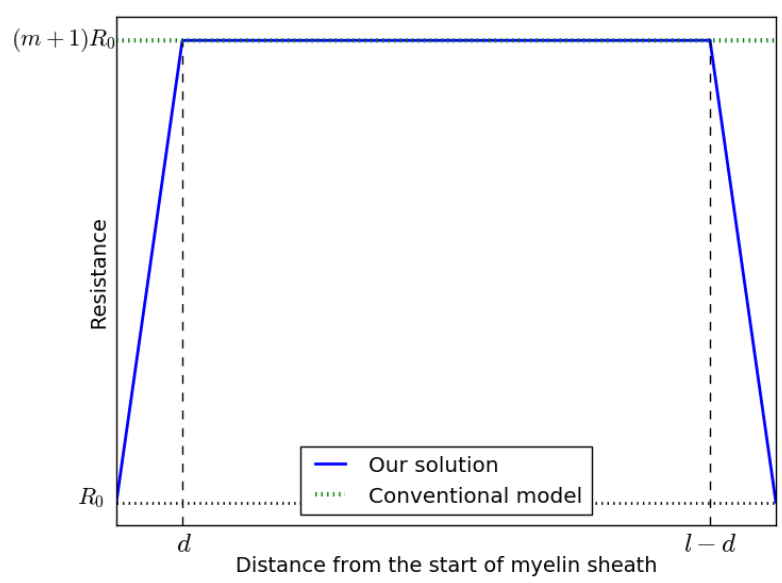

Fig. 4 - Leakage resistance trend between two Ranvier nodes.

As we can see in Fig. 4, the resistance gradually increases in the paranodal region, from $R_{0}$, the resistance of naked axolemma, to $(m+1) R_{0}$, axolemma covered with $m$ layers of myelin, while the conventional model assumes it to be constant. For capacitance, however, the conventional model understates the capacitance, as can be seen in Fig. 5. The capacitance values drop from $C_{0}$ to $C_{0} /(m+1)$. In Fig. 4 and Fig. 5, $d$ marks the boundary of the first

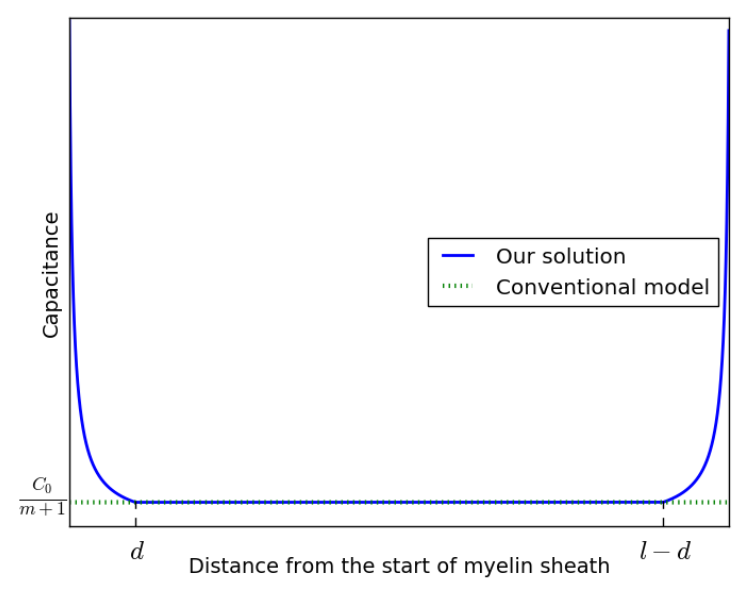

Fig. 5 - Leakage capacitance trend between two Ranvier nodes.

paranodal region, while $l-d$ marks the start of the end paranodal region.

Note that the effects of myelin layers on leakage resistance and leakage capacitance are the same because leakage capacitance being inversely proportional to the capacitance value while leakage resistance being linearly proportional to the resistance value.

\subsection{Frequency response analysis of internode}

In this section, we present the differences between our model and the conventional model. In this subsection, unless we are testing that specific variable, we use the simulation parameters given in Table 1 . The frequency responses are simulated for the frequency range of $0-$ $6000 \mathrm{~Hz}$.

First, we examine the attenuation depending on frequency over a single myelinated segment. The attenuation values are essential in determining the continuity of action potential through the whole axon because the voltage levels at the end of the segment determine whether the voltage-controlled channels in the Node of Ranvier are activated. Since the conventional method underestimates leakage in the paranodal regions by assuming a cylindrical myelin cover, the attenuation values are lower compared to our model. The frequency response of a single myelinated region is given in Fig. 6. Even though our solution traces a very similar path to the conventional method, the percent difference increases dramatically for higher frequency values, reaching $253 \%$ for $f=6000 \mathrm{~Hz}$. As a result of this extreme difference, propagation of spontaneous high-frequency spikes, a complication related to axonal injuries may be underestimated using the conventional method. Furthermore, the discrepancy is not negligible for $f<500 \mathrm{~Hz}$, where most of the spectrum of a normal action potential lies. Therefore, our model may correct the shape of a healthy action potential measured at the end of the myelinated segment. 
Table 1 - Model and simulation parameters

\begin{tabular}{l|c|c|c}
\hline Parameter & Value & Unit & Symbol \\
\hline \hline Specific forward resistance & $100[26]$ & $\Omega m$ & $r_{f}$ \\
\hline Specific leakage resistance & $10^{6}[26]$ & $\Omega m^{2}$ & $r_{l}$ \\
\hline Specific membrane leakage capacitance & $10^{-2}[26]$ & $F m^{-2}$ & $c_{m}$ \\
\hline Axolemma thickness & $5 \times 10^{-9}[27]$ & $m$ & $d_{m}$ \\
\hline Length of myelinated segment & $1 \times 10^{-3}[28]$ & $m$ & $l$ \\
\hline Length of paranodal region & $1 \times 10^{-4}[21]$ & $m$ & $d$ \\
\hline Axon radius & $10^{-5}[21]$ & $m$ & $a$ \\
\hline Myelin turn number & $20[29]$ & & $n$ \\
\hline
\end{tabular}

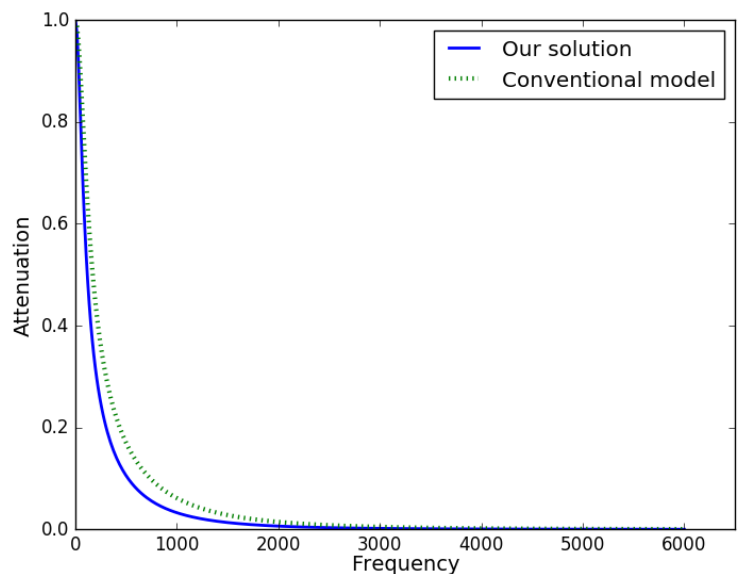

Fig. 6 - Attenuation vs frequency for a single myelinated segment.

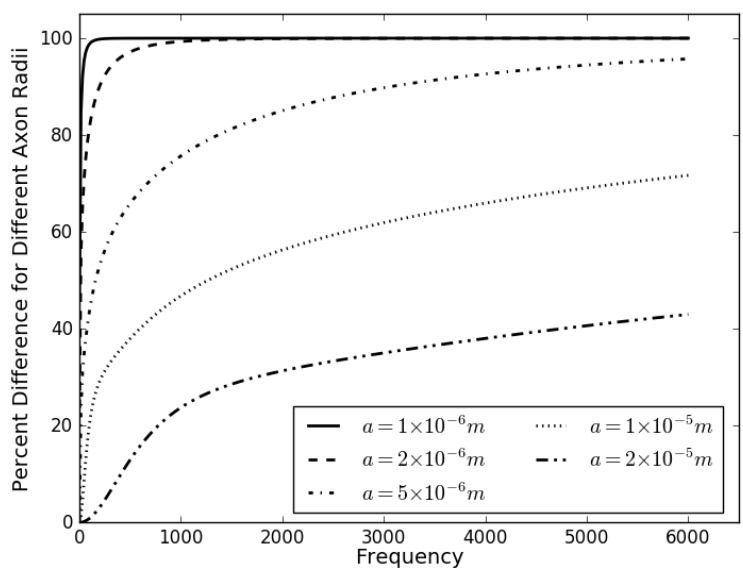

Fig. 7 - Percent difference between our model and conventional model for different frequency values depending on axon radii.

In Fig. 7, we show the percent difference for attenuation between our model and the conventional solution for different axon radii. For larger radii values, the effect of leakage is less so that our correction becomes insignificant. However, for typical and smaller radii values, our approach offers a dramatic correction over the conventional approach.

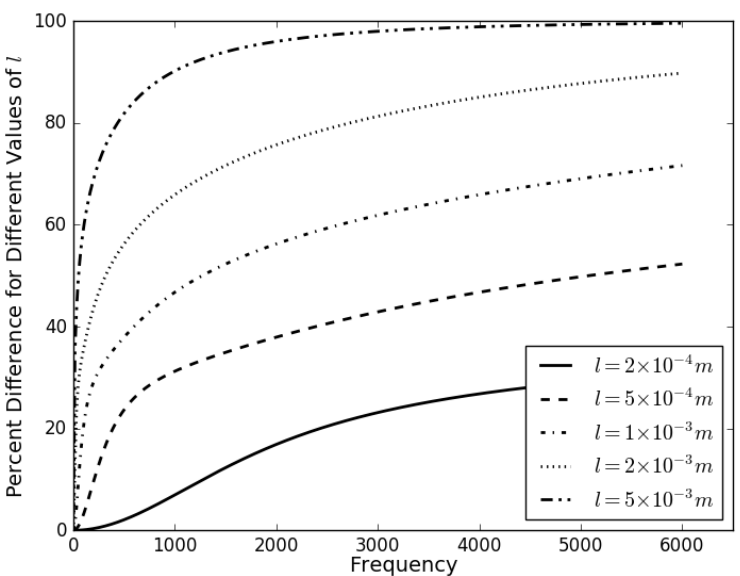

Fig. 8 - Percent difference between our model and conventional model for different myelin turn values.

In Fig. 8, we analyzed the percent difference between our model and the conventional solution in attenuation for different lengths of myelinated segments. Note that here we assumed the length of the paranodal regions are of the same ratio. That is, for longer myelinated segments, the paranodal regions were also assumed to be longer. As the length of the segment decreases, the overall leakage diminishes. As a result, our correction becomes less significant. However, for typical lengths of myelinated segments, the correction offers a tremendous change over the conventional method, especially for high-frequency values.

In Fig. 9, we focused on the effect of changing the ratio over the paranodal region length, $d$ over the length of the whole myelinated segment, $l$. As expected, as the length of the paranodal segment diminishes, our correction becomes less significant. However, even for shorter paranodal regions, the correction may be important.

Fig. 10 analyzes the relationship between the maximum myelin cover, $n$, and the percent difference between our solution and the conventional cable theory approach. As expected, for low myelin cover, the paranodal regions are essentially similar to the rest of the segment. Thus, our correction becomes insignificant, especially for the lowfrequency region. 


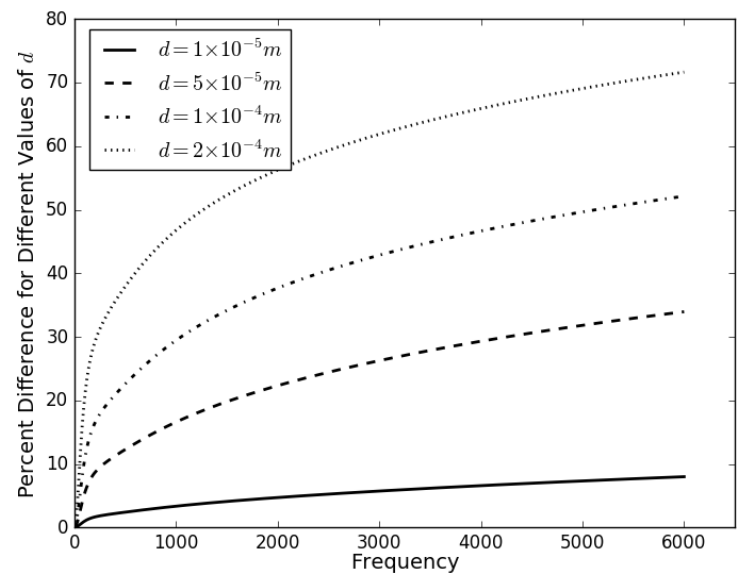

Fig. 9 - Percent difference between our model and conventional model for different frequency values depending on the length of the paranodal region.

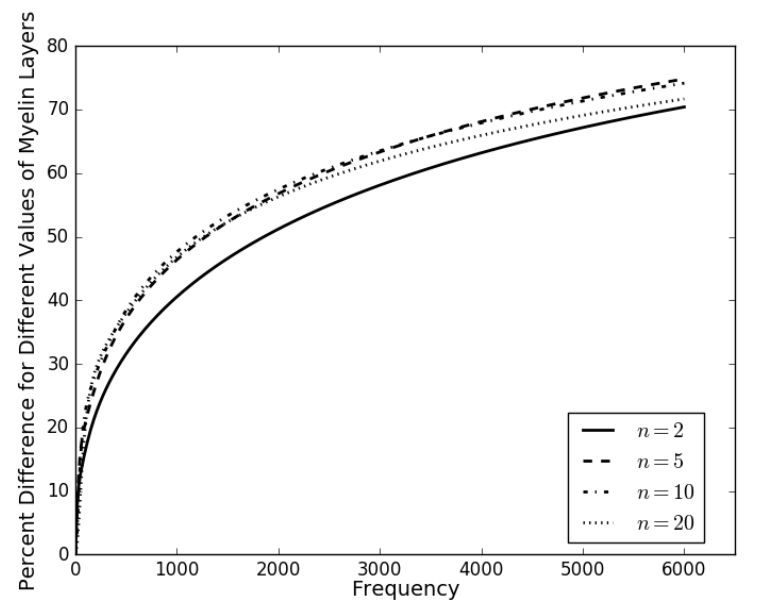

Fig. 10 - Percent difference between our model and conventional model for different frequency values depending on axon length.

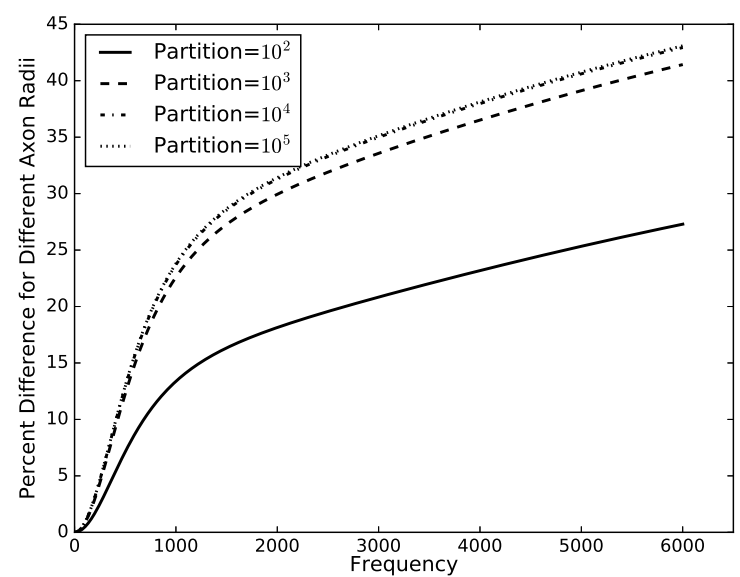

Fig. 11 - Percent difference between our model and conventional model for different step size values used for finite element analysis.
Fig. 11 shows the percent difference between our model and the conventional solution for different partition numbers. For the results presented above, we partitioned a single myelinated segment into $10^{4}$ compartments. Note that, in Fig. 11, the percent difference values for $10^{4}$ compartments and $10^{5}$ compartments are indistinguishable, proving that our finite element method analysis has sufficient depth to offer accurate results, i.e.,

$$
G_{\Delta x=\frac{l}{10^{4}}} \approx G_{\Delta x=\frac{l}{10^{5}}} \approx \lim _{\Delta x \rightarrow 0} G_{\Delta x} .
$$

\section{CONCLUSION AND FUTURE DIRECTIONS}

In this work, we developed a compartment-based model for the endpoints of the myelin sheath, i.e., the paranodal region, for the first time in literature. We believe our work paves the way to more complicated and realistic models to apply ICT-based treatments to conditions arising from deformities in the myelin sheath.

In future work, several issues will be investigated as follows:

- Incorporating nodes of Ranvier into the model to obtain end-to-end axonal channel characteristics,

- Extensive examination of axon's end-to-end model including delay and phase analysis,

- Calculation of digital and analog capacity for the axonal internodes,

- Modeling myelin-related diseases, e.g., paranodal detachment and demyelination, and determining the loss in internodal channel capacity due to a disease.

\section{ACKNOWLEDGEMENT}

This work was supported in part by the AXA Research Fund (AXA Chair for Internet of Everything at Koç University), ERC project MINERVA (ERC-2013-CoG \#616922), and Huawei Graduate Research Scholarship.

\section{REFERENCES}

[1] IF Akyildiz, M Pierobon, S Balasubramaniam, and Y Koucheryavy. "The internet of bio-nano things". In: IEEE Communications Magazine 53.3 (2015), pp. 32-40.

[2] Ozgur B Akan, Hamideh Ramezani, Tooba Khan, Naveed A Abbasi, and Murat Kuscu. "Fundamentals of molecular information and communication science". In: Proceedings of the IEEE 105.2 (2017), pp. 306-318.

[3] Caglar Koca and Ozgur B Akan. "Anarchy versus cooperation on internet of molecular things". In: IEEE Internet of Things Journal 4.5 (2017),

pp. 1445-1453. 
[4] Tooba Khan, Meltem Civas, Oktay Cetinkaya, Naveed A Abbasi, and Ozgur B Akan. "Nanosensor networks for smart health care". In: Nanosensors for Smart Cities. Elsevier, 2020, pp. 387-403.

[5] Meltem Civas and Ozgur B Akan. "Rate of Information Flow Across Layered Neuro-Spike Network in the Spinal Cord". In: IEEE transactions on nanobioscience 19.3 (2020), pp. 368-377.

[6] Ozgur Baris Akan, Hamideh Ramezani, Meltem Civas, Oktay Cetinkaya, Bilgesu Arif Bilgin, and Naveed Ahmed Abbasi. "Information and communication theoretical understanding and treatment of spinal cord injuries: State-of-the-art and research challenges". In: IEEE Reviews in Biomedical Engineering (2021).

[7] Caglar Koca, Meltem Civas, Selin M Sahin, Onder Ergonul, and Ozgur B Akan. "Molecular Communication Theoretical Modeling and Analysis of SARSCoV2 Transmission in Human Respiratory System". In: IEEE Transactions on Molecular, Biological and Multi-Scale Communications (2021).

[8] Mladen Veletić, Pål Anders Floor, Zdenka Babić, and Ilangko Balasingham. "Peer-to-peer communication in neuronal nano-network". In: IEEE Transactions on Communications 64.3 (2016), pp. 11531166.

[9] Eren Balevi and Ozgur B Akan. "A physical channel model for nanoscale neuro-spike communications". In: IEEE Transactions on Communications 61.3 (2013), pp. 1178-1187.

[10] Derya Malak and Ozgur B Akan. "A communication theoretical analysis of synaptic multipleaccess channel in hippocampal-cortical neurons". In: IEEE Transactions on communications 61.6 (2013), pp. 2457-2467.

[11] Hamideh Ramezani and Ozgur B Akan. "A Communication Theoretical Modeling of Axonal Propagation in Hippocampal Pyramidal Neurons". In: IEEE Transactions on NanoBioscience (2017).

[12] Alireza Khodaei and Massimiliano Pierobon. "An intra-body linear channel model based on neuronal subthreshold stimulation". In: Communications (ICC), 2016 IEEE International Conference on. IEEE. 2016, pp. 1-7.

[13] Peter Dayan and Laurence F Abbott. Theoretical neuroscience. Vol. 806.

[14] R. Shi and W. Sun. "Potassium channel blockers as an effective treatment to restore impulse conduction in injured axons". In: Neuroscience Bulletin 27.1 (2011), pp. 36-44.

[15] A. Ochab-Marcinek, G. Schmid, I. Goychuk, and P. Hänggi. "Noise-assisted spike propagation in myelinated neurons". In: Physical Review E 79.1 (2009), pp. 011904-1-7.
[16] C. F. Babbs and R. Shi. "Subtle Paranodal Injury Slows Impulse Conduction in a Mathematical Model of Myelinated Axons". In: PLOS ONE 8.7 (2013), pp. 1-11.

[17] M. N. Rasband and J. S. Trimmer. "Developmental clustering of ion channels at and near the node of Ranvier". In: Developmental Biology 236.1 (2001), pp. 5-16.

[18] Wilfrid Rall. "Core conductor theory and cable properties of neurons". In: Comprehensive physiology (2011).

[19] O. N. Favorsky. Thermal to Mechanical Energy Conversion : Engines and Requirements - Volume I. Paris: Encyclopedia of Life Support Systems (EOLSS) Publishers, 2009.

[20] F. Gabbiani and S. Cox. Mathematics for Neuroscientists. London: Academic Press, 2010.

[21] Charles F Babbs and Riyi Shi. "Subtle paranodal injury slows impulse conduction in a mathematical model of myelinated axons". In: PLoS One 8.7 (2013), e67767.

[22] A Aldo Faisal, Luc PJ Selen, and Daniel M Wolpert. "Noise in the nervous system". In: Nature reviews. Neuroscience 9.4 (2008), p. 292.

[23] Amit Manwani and Christof Koch. "Detecting and estimating signals in noisy cable structures, I: Neuronal noise sources". In: Neural computation 11.8 (1999), pp. 1797-1829.

[24] A Aldo Faisal and Simon B Laughlin. "Stochastic simulations on the reliability of action potential propagation in thin axons". In: PLoS computational biology 3.5 (2007), e79.

[25] John G Proakis and Masoud Salehi. Digital Communications 5e. 2008.

[26] Ryosuke Matsumura, Hideaki Yamamoto, Takeshi Hayakawa, Shutaro Katsurabayashi, Michio Niwano, and Ayumi Hirano-Iwata. "Dependence and homeostasis of membrane impedance on cell morphology in cultured hippocampal neurons". In: Scientific reports 8.1 (2018), pp. 1-10.

[27] Srikantan S Nagarajan and Dominique M Durand. "Analysis of magnetic stimulation of a concentric axon in a nerve bundle". In: IEEE Transactions on Biomedical Engineering 42.9 (1995), pp. 926-933.

[28] Keiichiro Susuki. "Myelin: a specialized membrane for cell communication". In: Nature Education 3.9 (2010), p. 59.

[29] Nicolas Snaidero and Mikael Simons. "Myelination at alance". In: Journal of cell science 127.14 (2014), pp. 2999-3004. 


\section{AUTHORS}

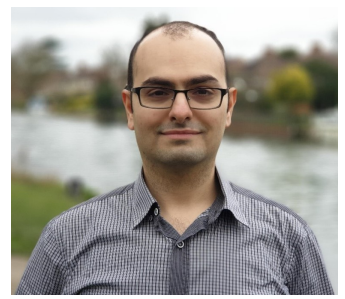

Caglar Koca received a B.Sc. degree in electrical and electronics engineering from Middle East Technical University, Ankara, Turkey, in 2010, an M.Sc. degree in electrical and electronics engineering from Koc University, Istanbul, Turkey, in 2014, and is currently pursuing a Ph.D. degree at University of Cambridge. He joined the Next-Generation and Wireless Communication Laboratory (NWCL), Koc University in 2011 and he is currently a research assistant at the Internet of Everything (IoE) group, University of Cambridge since 2017. His research interests include Internet of Everything, Information Theory and molecular communications.

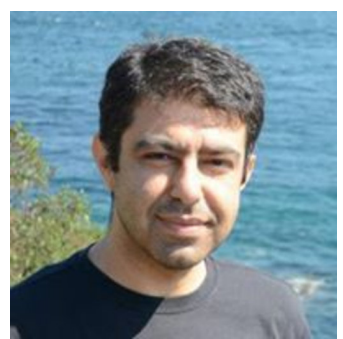

Ozgur Ergul received his M.S. degree from Middle East Technical University, Turkey, and Ph.D. degree from the NextGeneration Communication Laboratory, Koç University, Turkey respectively, both in electrical and electronics engineering. He is currently with the Department of Electrical and Electronics Engineering, Gazi University, Faculty of Engineering, Turkey. His current research interests include vehicular networks, visible light communications, and the Internet of Things.

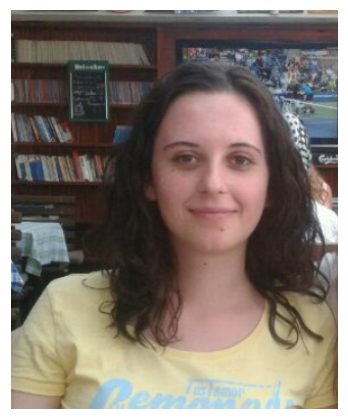

Meltem Civas received her B.S. degree in Electrical and Electronics Engineering in Bilkent University, Ankara, Turkey, in January 2016, and her M.S. degree at the Electrical and Electronics Engineering Department, Koç University, Istanbul, Turkey in September 2018. She is currently a research assistant at the Next-generation and Wireless Communications Laboratory and pursuing her Ph.D. degree in Electrical and Electronics Engineering, Koç University. Her research interests include Internet of Everything and Molecular Communications.

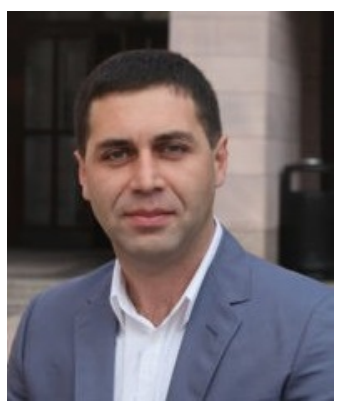

Ozgur B. Akan received his Ph.D. degree in electrical and computer engineering from the Broadband and Wireless Networking Laboratory, School of Electrical and Computer Engineering, Georgia Institute of Technology, Atlanta in 2004. He is currently with the Electrical Engineering Division, Department of Engineering, University of Cambridge, United Kingdom, and also the director of the Next-Generation and Wireless Communications Laboratory in the Department of Electrical and Electronics Engineering, Koç University. His research interests include wireless, nano, and molecular communications, and the Internet of Everything. 